BMJ Open

Sport \&

Exercise

Medicine

\title{
Decrease in eccentric quadriceps and hamstring strength in recreational alpine skiers after prolonged skiing
}

\author{
Arnold Koller, Birgit Fuchs, Veronika Leichtfried, Wolfgang Schobersberger
}

To cite: Koller A, Fuchs B, Leichtfried V, et al. Decrease in eccentric quadriceps and hamstring strength in recreational alpine skiers after prolonged skiing. BMJ Open Sport Exerc Med 2015;0: e000028. doi:10.1136/ bmjsem-2015-000028

- Prepublication history for this paper is available online To view these files please visit the journal online (http://dx.doi.org/10.1136/ bmjsem-2015-000028).

Accepted 23 July 2015

\section{ABSTRACT}

Background: To effectively prevent injury in recreational alpine skiing, it is important to identify modifiable risk factors that can be targeted through exercise and training. Fatigue is a potential risk factor in recreational skiing, but no investigations have evaluated concentric/eccentric quadriceps and hamstring fatigue in recreational skiers. We tested the hypothesis that recreational skiing is associated with more pronounced eccentric as compared with concentric muscle fatigue.

Methods: Twenty-four healthy and fit recreational skiers (14 male and 10 female) performed an isokinetic muscle test 1 day before, $1 \mathrm{~h}$ after, and $24 \mathrm{~h}$ after a $4 \mathrm{~h}$ skiing session. The testing protocol consisted of concentric and eccentric quadriceps and hamstring contractions for both legs.

Results: Eccentric peak hamstring torque (both thighs) and eccentric peak quadriceps torque (left thigh) were reduced in male and female participants $(p<0.05)$. Reduced peak torques were still present $24 \mathrm{~h}$ after the skiing session. There were no other significant findings.

Summary: Recreational skiing is associated with prolonged (at least $24 \mathrm{~h}$ ) eccentric quadriceps (left thigh) and hamstring (both thighs) fatigue in men and women. Eccentric quadriceps and hamstring fatigue may be a potential injury risk factor in male and female recreational skiers. This provides some justification for judicious use of additional eccentric training modalities for alpine skiing.

Changes in modern ski equipment and improvements in slope design and maintenance have contributed to a decline in injuries, yet there is still a significant number of skiing injuries. ${ }^{1}$ Previously, fractures were more common, but since these technical changes, injuries of the ligaments of the knee have increased. ${ }^{2}$

To prevent injury effectively, we should identify modifiable risk factors that can be targeted through exercise and training. ${ }^{3}$ According to expert stakeholders in the World Cup ski racing community, a superior fitness level is one of the most important

\section{Summary box}

- This study is the first to show that recreational skiing is associated with prolonged eccentric quadriceps and hamstring fatigue.

- Presumably, concentric types of endurance training (eg, biking, walking) do not prevent fatigue during eccentric (ie, skiing) types of endurance exercise.

- Judicious use of eccentric types of endurance training may be warranted in recreational skiers.

perceived factors for injury prevention. ${ }^{4}$ Although the importance of a high-fitness level for injury prevention is widely accepted in practice, this is not supported by scientifically validated recommendations. ${ }^{3}$

Fatigue is known to have a negative impact on balance control, and physical fitness has a positive effect on reaction time during exercise. ${ }^{56}$ Therefore, it seems reasonable that a lack of fitness and early fatigue could both be independent risk factors for injuries in alpine skiers. ${ }^{7}$ Surprisingly, perceived fatigue does not seem to be a major risk factor for anterior cruciate ligament (ACL) injury in recreational skiers. ${ }^{8}$ Moreover, eccentric knee extensor muscle activity is considered a particular feature of alpine skiing and the preventive exercises deemed most important for preventing injuries in alpine skiing are eccentric exercises (eg, eccentric training to shift the optimal muscle length of hamstring muscles). ${ }^{9}$ In contrast to competitive alpine skiing, a significantly higher risk of injury has been reported in women than men in recreational skiing. ${ }^{10}$ During turns in alpine skiing, eccentric knee extensor muscle activity is dominant and is higher in magnitude and up to twice as long in duration compared with concentric activity. ${ }^{9}$ Unexpectedly, mitochondrial protein concentration does not increase in the vastus lateralis muscle after 28.5 days of alpine skiing over 3 months. ${ }^{11}$ In contrast, an 
increased capillary-to-fibre ratio and capillary density reflects a net increase in capillary supply to knee extensor muscles after ski training, and adaptations of muscle energy supply lines with recreational skiing differ from the effects of concentric types of endurance training. ${ }^{11}$ This finding is consistent with a specific increase in capillarity with eccentric types as opposed to concentric types of endurance exercise, ${ }^{12}$ suggesting that modifications in capillarity with skiing are driven by mechanical rather than metabolic factors. ${ }^{11}$ Moreover, eccentric hamstring fatigue occurs in response to football-specific exercise and marathon running. ${ }^{13}{ }^{14}$ However, to the best of our knowledge, no investigations have aimed at evaluating concentric/eccentric quadriceps and hamstring fatigue in alpine skiing. Thus, we aimed to test the hypothesis that recreational alpine skiing is associated with pronounced eccentric muscle fatigue.

\section{METHODS}

Study design

The recruitment process for this study involved broadcasting via local radio stations. To obtain a homogeneous sample, middle-aged participants were selected according to their skiing ability and physical activity. All participants were at an intermediate level based on the Austrian Ski Teaching Concept. ${ }^{15}$ Intermediate-level skiers are able to perform short-radii and long-radii turns on prepared terrains. On flat terrain, intermediate-level skiers are able to execute carved turns but perform mostly skid turns on steep terrain. Physical activity in leisure time was graded as one of four levels using the Copenhagen City Heart Study Leisure Time Physical Activity Questionnaire. ${ }^{16}$ Participants belonged to group III: light physical activity for more than $4 \mathrm{~h}$ per week or more vigorous activity for 2-4 h per week (eg, brisk walking, fast biking, sports that cause perspiration or exhaustion). ${ }^{16}$ The activity questionnaire has been shown to discriminate between sedentary people and their more active counterparts with respect to maximal oxygen uptake. ${ }^{16}$ No systematic eccentric-based training protocols were implemented in the training programmes. Thirty skiers volunteered to participate in this study.

All participants underwent the following screening protocol. First, after obtaining the medical history and conducting a physical examination, a 12-lead electrocardiogram at rest and spirometry were performed. In addition, blood was collected, and standard laboratory parameters (including red and white cell counts and C reactive protein levels) were measured. Participants were excluded if they suffered from major chronic illnesses such as hypertension, diabetes, chronic obstructive pulmonary disease, coronary artery disease requiring treatment or had a history of knee joint ligamentous trauma. An incremental standard cycle protocol was used to determine peak exercise capacity.

One day before the skiing session, 24 participants (14 male and 10 female skiers) performed an isokinetic muscle test. The test was repeated $1 \mathrm{~h}$ and 1 day after the skiing session. The dominant leg was determined by asking skiers which leg they prefer to kick a ball with, as described by another study. ${ }^{17}$ Written informed consent was given by all participants. The study protocol was approved by the ethics committee of the Medical University of Innsbruck (AN-2013-0028).

\section{Cycle ergometry}

Participants' performance capacity was assessed by maximal exercise testing on the Excalibur Sport cycle ergometer (Lode B.V., Groningen, the Netherlands). Male participants started at $70 \mathrm{~W}$, with stepwise increases of $30 \mathrm{~W}$ every $2 \mathrm{~min}$ until exhaustion. Female participants started at $50 \mathrm{~W}$, with stepwise increases of $25 \mathrm{~W}$ every $90 \mathrm{~s}$. Capillary blood samples were taken from the ear lobe immediately after each workload to determine lactate concentration (lactateanalyser Biosen C_line, EKF Diagnostic, Barleben, Germany). Values of maximal power output during cycle ergometry corrected for body mass were used to assess aerobic power. ${ }^{18}$

\section{Isokinetic dynamometry}

An isokinetic dynamometer (CON-TREX MJ; CMV AG, Zürich, Switzerland) was used to generate angle-torque curves. An angle-torque curve is a measure of torque as a function of knee angle produced when the muscle is maximally activated during isovelocity shortening or stretching. After warming up for $10 \mathrm{~min}$ on a bicycle, participants sat on the dynamometer with their hip flexed to approximately $90^{\circ}$ and their trunk secured with dual cross-over straps and a waist strap. The range of motion at the knee was set at $110^{\circ}$ and was modified according to the available passive motion and the participant's tolerance. The angle was $0^{\circ}$ when the leg was fully extended at the knee and $110^{\circ}$ when it was flexed. A thigh strap on the test leg was used to restrict any lateral movement at the knee, allowing only flexion and extension. Both legs were tested, and the testing protocol consisted of concentric and eccentric quadriceps and hamstring contractions (ie, four repetitions each at an angular velocity of $60^{\circ} / \mathrm{s}$ ) after familiarisation with the equipment (2 min; submaximal trials at an angular velocity of $60^{\circ} / \mathrm{s}$ ). Torque and angle signals were transferred from the dynamometer to a computer and analysed. Optimum peak torque was recorded for hamstrings and quadriceps of both legs and used for statistical analyses. In addition, optimum peak torque was corrected for body mass.

\section{Skiing}

The prolonged skiing session took place at the Patscherkofel Ski Area in Innsbruck, Austria. The testing period was in March 2014. Participants were requested to ski at their habitual speed using their preferred technique; thus, they skied at their own self-selected pace. Weather conditions were similar for all participants (ie, no snowfall). The skiing session started at about 9:00. 
After a 60 min break following $2 \mathrm{~h}$ of skiing, participants skied for an additional $2 \mathrm{~h}$. With GPS tracking (iSki Tracker, Feratel Media Technologies AG, Innsbruck, Austria), slope kilometres, vertical distance (ie, metres of altitude) and speed were recorded. Heart rate was measured continuously via the Polar RS 400 (Polar Electro Oy, Kempele, Finland) during the entire skiing session.

\section{Statistical analysis}

SPSS V.20.0 (Statistical Package for Social Sciences, Chicago, Illinois, USA) was used to perform all statistical analyses. The distribution of data was evaluated using box plots. All parameters were normally distributed. Following significant effects in repeated measures analysis of variance, Student's t tests were used to identify significant changes in parameters between each time point and baseline values. Student's t tests were also used to analyse group differences. Categorical variables are reported as proportions, and numerical data are reported as mean \pm SD. A $p$ value of $<0.05$ was considered statistically significant.

\section{RESULTS}

After physical examination and ergometry, six participants were excluded (two men and four women). Therefore, 24 participants (14 males and 10 females) underwent further testing. Age, height and weight of the remaining 24 participants were $49.0 \pm 6.7$ years, 172.3 $\pm 10.1 \mathrm{~cm}$ and $73.3 \pm 12.5 \mathrm{~kg}$, respectively. All participants finished the skiing session. Maximum heart rate (141.2 $\pm 13.4 \mathrm{bpm}$ ) during skiing was $77.4 \%$ of the maximum heart rate measured during the incremental cycle test $(182.4 \pm 9.8 \mathrm{bpm})$. Interestingly, there were no significant differences in peak torque before and after skiing, except for eccentric peak hamstring torque (both thighs) and eccentric peak quadriceps torque (left thigh) (table 1).

Additional evaluation of sex differences showed that the right leg was the most preferred kicking leg in $86 \%$ of men and $80 \%$ of women. Values of maximal power output corrected for body mass were significantly different between women $(2.92 \pm 0.39 \mathrm{~W} / \mathrm{kg})$ and men $(3.3$ $\pm 0.59 \mathrm{~W} / \mathrm{kg}) \quad(\mathrm{p}=0.003)$. Baseline absolute peak torque values (concentric and eccentric) and baseline concentric peak torque values corrected for body mass were also significantly different between female and male skiers (table 2).

In contrast, female and male skiers did not display significant differences in eccentric peak torque corrected for body mass, except that eccentric peak hamstring torque corrected for body mass was reduced in the right thigh of females (table 2). Moreover, male and female skiers did not display significant bilateral limb differences in thigh muscle strength for any of the strength variables (table 2). No significant differences were observed in slope kilometres between females $(31.1 \pm 4.8 \mathrm{~km})$ and males $(30.3 \pm 5.8 \mathrm{~km})$. Maximal speed during skiing was significantly higher in male skiers $(77.5 \pm 17.4 \mathrm{~km} / \mathrm{h})$ than in female skiers $(63.4 \pm 7.05 \mathrm{~km} / \mathrm{h})$. Only eccentric peak hamstring torque (both thighs) and peak quadriceps torque (left thigh) were significantly reduced in females and males after $4 \mathrm{~h}$ of skiing. In addition, women and men did not display significant differences in change in peak torques $(\triangle \mathrm{PT})$ for any of the strength variables (ps $>0.05$ ), except for eccentric peak hamstring torque $\left(\triangle \mathrm{PTH} \mathrm{ecc}_{\mathrm{e}}\right)$ (left thigh) 1 day after the ski session (males: $\Delta \mathrm{PTH}_{\mathrm{ecc}}=1.2 \mathrm{~N} \mathrm{~m}$ (1\% decline); females: $\Delta \mathrm{PTH}_{\mathrm{ecc}}=9.7$ $\mathrm{N} \mathrm{m}$ (11.6\% decline); $\mathrm{p}=0.013)$. No significant differences in change in eccentric peak quadriceps torque corrected for body mass $\left(\triangle \mathrm{PTQ}(\mathrm{BW})_{\mathrm{ecc}}\right)$ (left thigh) were observed in either women $\left(\triangle \mathrm{PTQ}(\mathrm{BW})_{\mathrm{ecc}}=0.19 \pm 0.34(1 \mathrm{~h}\right.$

Table 1 Peak hamstring and quadriceps torque in recreational skiers after $4 \mathrm{~h}$ of skiing

\begin{tabular}{|c|c|c|c|}
\hline \multirow[b]{2}{*}{ Muscle } & \multicolumn{3}{|c|}{ Peak torque (N m) } \\
\hline & Before skiing & $1 \mathrm{~h}$ after skiing & $24 \mathrm{~h}$ after skiing \\
\hline \multicolumn{4}{|l|}{ Hamstring } \\
\hline \multicolumn{4}{|c|}{ Concentric contractions } \\
\hline Right thigh & $78.4 \pm 24.1$ & $79.3 \pm 24.0$ & $78.3 \pm 22.5$ \\
\hline Left thigh & $77.9 \pm 22.8$ & $78.7 \pm 24.6$ & $82.4 \pm 26.2$ \\
\hline \multicolumn{4}{|c|}{ Eccentric contractions } \\
\hline Right thigh & $103.9 \pm 33.2$ & $98.3 \pm 31.2^{*}$ & $93.8 \pm 29.1^{*}$ \\
\hline Left thigh & $105.8 \pm 31.6$ & $101.1 \pm 36.0$ & $96.8 \pm 29.1^{*}$ \\
\hline \multicolumn{4}{|c|}{ Quadriceps } \\
\hline \multicolumn{4}{|c|}{ Concentric contractions } \\
\hline Right thigh & $131.4 \pm 41.8$ & $128.6 \pm 39.9$ & $120.6 \pm 43.7$ \\
\hline Left thigh & $138.2 \pm 44.4$ & $133.0 \pm 41.6$ & $132.9 \pm 42.3$ \\
\hline \multicolumn{4}{|c|}{ Eccentric contractions } \\
\hline Right thigh & $178.3 \pm 50.8$ & $171.1 \pm 44.6$ & $172.1 \pm 45.5$ \\
\hline Left thigh & $176.1 \pm 48.0$ & $164.8 \pm 52.4^{*}$ & $164.6 \pm 47.7^{\star}$ \\
\hline
\end{tabular}


Table 2 Comparison of absolute (PT) and body weight-normalised (PT/BW) baseline peak torque between male and female skiers

\begin{tabular}{|c|c|c|c|c|}
\hline \multirow[b]{2}{*}{ Muscle } & \multicolumn{2}{|l|}{ Males } & \multicolumn{2}{|l|}{ Females } \\
\hline & PT (N m) & PT/BW (N m/kg) & PT (N m) & PT/BW (N m/kg) \\
\hline \multicolumn{5}{|l|}{ Hamstring } \\
\hline Right thigh & $91.9 \pm 20.1$ & $1.16 \pm 0.23$ & $59.6 \pm 15.0^{*}$ & $0.93 \pm 0.24^{*}$ \\
\hline Left thigh & $92.4 \pm 16.8$ & $1.17 \pm 0.18$ & $57.6 \pm 11.8^{*}$ & $0.90 \pm 0.20^{*}$ \\
\hline \multicolumn{5}{|c|}{ Eccentric contractions } \\
\hline \multicolumn{5}{|c|}{ Quadriceps } \\
\hline \multicolumn{5}{|c|}{ Concentric contractions } \\
\hline Right thigh & $155.1 \pm 35.0$ & $1.96 \pm 0.40$ & $98.2 \pm 24.5^{\star}$ & $1.54 \pm 0.40^{\star}$ \\
\hline Left thigh & $164.6 \pm 36.5$ & $2.07 \pm 0.34$ & $101.1 \pm 22.2^{*}$ & $1.56 \pm 0.28^{\star}$ \\
\hline \multicolumn{5}{|c|}{ Eccentric contractions } \\
\hline
\end{tabular}

after skiing) and $0.14 \pm 0.21$ (24 h after skiing)) or men $\left(\triangle \mathrm{PTQ}(\mathrm{BW})_{\mathrm{ecc}}=0.13 \pm 0.29 \quad(1 \mathrm{~h}\right.$ after skiing) and 0.13 \pm 0.31 (24 h after skiing) $\quad(\mathrm{p}=0.65$ ( $1 \mathrm{~h}$ after skiing); $\mathrm{p}=0.92(24 \mathrm{~h}$ after skiing $))$.

\section{DISCUSSION}

Different methodological approaches in recent years have increased our understanding of why and how injuries occur in alpine skiing. ${ }^{19}$ However, there is a paucity of scientific literature on modifiable (ie, trainable) risk factors for injury prevention in recreational skiers. ${ }^{3}$ In nonprofessional skiing, female skiers have twice the risk of serious knee injuries as male skiers. ${ }^{2}$ Environmental factors, such as cold temperature and snowfall, as well as personal factors, such as leg dominance, could potentially have a greater impact on injury risk among women. ${ }^{17} 20$

\section{Limb strength after prolonged skiing}

The current study did not involve an extreme skiing load. The participants performed at an intensity that is consistent with recreational skiing. ${ }^{21}$ We found prolonged (ie, at least 1 day) eccentric hamstring fatigue in thighs and eccentric quadriceps fatigue in the left thigh in recreational endurance-trained (ie, concentric type of endurance exercise ${ }^{11}$ ) alpine skiers even at the end of the winter season, when participants may be conditioned to eccentric loading during skiing. ${ }^{9}$ Consequently, one might expect a more pronounced decline in eccentric strength at the start of the winter season. This suggests eccentric training as a training adjunct in strength and conditioning programmes for recreational alpine skiers.

\section{Eccentric fatigue and perceived exertion}

A recent study reported that ACL-injured recreational skiers perceive significantly less local and overall fatigue than uninjured controls, ${ }^{8}$ although there were some limitations of the interview approach used in this study. Perceived exertion is reported to be 'fairly light' during eccentric exercise. $^{22} 23$ Consequently, perceived fatigue may not reflect true fatigue (ie, decline in eccentric muscle strength) during eccentric exercises, challenging the notion that ACL injuries may not be related to fatigue. ${ }^{8}$

\section{Comparison of limb strength between male and female skiers}

Baseline values of absolute strength were lower in female skiers than in male skiers. The use of absolute strength values, however, may not be appropriate when comparing men and women. Instead, body mass normalisation is recommended. ${ }^{2425}$ Despite there being no significant difference in concentric peak torque before and after skiing, and no difference in slope kilometres skied between men and women, concentric peak torque values corrected for body mass and body weightnormalised maximal power output during cycle ergometry were significantly lower in female skiers than in male skiers. This suggests that concentric muscle action may not be a performance-limiting factor in prolonged recreational skiing. This is hardly surprising, because during recreational skiing an eccentric type as opposed to a concentric type of endurance exercise is dominant. ${ }^{11}$ Interestingly, previous work shows no correlation between racing performance and concentric peak torque and work for knee extension and flexion in either sex of elite skiers. ${ }^{26}$ Thus, one may speculate that concentric muscle action may also not be a performance-limiting factor in ski racing. ${ }^{27}$

Strikingly, there was no sex difference in baseline body weight-normalised eccentric peak quadriceps torque. Although female skiers showed lower eccentric peak 
hamstring torque corrected for body mass (right thigh) compared with male skiers, both sexes displayed reduced eccentric hamstring strength in both thighs after skiing. Interestingly, a recent study by Jordan $e t a l^{5}$ reports no sex differences in hamstring or quadriceps strength measured using isometric dynamometry when corrected for body mass in ski racers. In addition, no bilateral limb difference in thigh muscle strength was detected between male and female skiers. For knee/ ACL injuries, leg dominance could potentially have a higher impact on injury risk in women than in men. ${ }^{17}$ This study shows that eccentric quadriceps fatigue occurs in the left (ie, non-dominant) leg in male and female skiers. This decline in eccentric quadriceps strength in the non-dominant thigh may thus have the same impact on injury risk in both sexes.

\section{Eccentric hamstring fatigue and alpine skiing}

In a recent study, the effect of prolonged skiing on the recruitment and coordination of muscle activity was investigated in recreational skiers using electromyography (EMG). ${ }^{21}$ Interestingly, functional demand for the rectus femoris (RF) muscle during skiing, assessed by a wavelet analysis of EMG, is very high. ${ }^{21} 28$ The decline of eccentric hamstring strength measured in this study is consistent with this high functional demand for the RF during prolonged skiing. ${ }^{21}$ Moreover, this decline is consistent with the reduced eccentric hamstring force found in response to football-specific exercise and marathon running. ${ }^{13}{ }^{14}$ However, despite the considerable importance placed on hamstring eccentric exercises in premier league football teams, ${ }^{29}$ the amount of scientific evidence for this being an independent risk factor is low. ${ }^{30}$ This does not imply that this perception and practice is not valid or important; rather, there may simply not be a sufficient number of studies to validate or refute this practice. ${ }^{30}$ The hamstring muscles act as an ACL agonist to resist anterior translation of the tibia relative to the femur, ${ }^{31}{ }^{32}$ which is relevant for ACL injury prevention in alpine skiing. Moreover, the hamstring and quadriceps muscles act in a coordinated manner for knee stabilisation and ACL protection. ${ }^{31-33}$ Therefore, it seems reasonable that the decline in eccentric hamstring strength could be a risk factor for injuries in alpine skiing. However, as for football, ${ }^{30}$ there is currently no scientific evidence for this possibility. ${ }^{3}$ Nevertheless, the judicious use of hamstring eccentric training modalities may be warranted in the preparation of recreational skiers. ${ }^{9}$

\section{Limitations}

Some limitations of the present investigation include its relatively small sample size. Also, future studies should be undertaken to confirm the present results in younger and older skiers and to test whether eccentric fatigue lasts more than 1 day. Considering the current findings and the importance that sports medicine and sports science practitioners place on eccentric exercise in the preparation of alpine skiers, ${ }^{9}$ future research should determine the contribution of eccentric exercises to preventing injuries in this setting.

Contributors This study was conceived and designed by AK, BF and WS. Literature search was performed by AK. Acquisition of data and statistical analysis were carried out by $\mathrm{AK}, \mathrm{BF}$ and $\mathrm{VL}$, respectively. All authors have significantly contributed to this work. All authors contributed to the interpretation and discussion of the findings and participated in editing or rewriting of the article.

Competing interests None declared.

Ethics approval Ethics committee of the Medical University of Innsbruck.

Provenance and peer review Not commissioned; internally peer reviewed.

Open Access This is an Open Access article distributed in accordance with the Creative Commons Attribution Non Commercial (CC BY-NC 4.0) license, which permits others to distribute, remix, adapt, build upon this work noncommercially, and license their derivative works on different terms, provided the original work is properly cited and the use is non-commercial. See: http:// creativecommons.org/licenses/by-nc/4.0/

\section{REFERENCES}

1. Ruedl G, Philippe M, Sommersacher R, et al. Current incidence of accidents on Austrian ski slopes. Sportverl Sportschad 2014;28:183-7.

2. Palmer-Green D, Elliott N. Sports injury and illness epidemiology: Great Britain Olympic Team (TeamGB) surveillance during Sochi 2014 Winter Olympic Games. Br J Sports Med 2015;49:25-9.

3. Hébert-Losier K, Holmer H-C. What are the exercise-based injury prevention recommendations for recreational alpine skiing and snowboarding? A systematic review. Sports Med 2013;43:355-66.

4. Spörri J, Kröll J, Amesberger G, et al. Perceived key injury risk factors in World Cup alpine ski racing-an explorative qualitative study with expert stakeholders. Br J Sports Med 2012;46:1059-64.

5. Spörri J, Kröll J, Schwameder H, et al. Course setting and selected biomechanical variables related to injury risk in alpine ski racing: an explorative case study. Br J Sports Med 2012;46:1072-7.

6. Brisswalter J, Arcelin R, Audiffren M, et al. Influence of physical exercise on simple reaction time: effect of physical fitness. Percept Mot Skills 1997;85:1019-27.

7. Gilgien M, Spörri J, Kröll J, et al. Mechanics of turning and jumping and skier speed are associated with injury risk in men's World Cup alpine skiing: a comparison between the competition disciplines. $\mathrm{Br} \mathrm{J}$ Sports Med 2014;48:724-7.

8. Ruedl G, Schranz A, Fink C, et al. Are ACL injuries related to perceived fatigue in female skiers? JAI 2010;7.

9. Vogt $\mathrm{M}$, Hoppeler $\mathrm{HH}$. Eccentric exercise: mechanisms and effects when used as training regime or training adjunct. $J$ Appl Physiol 2014;116:1446-54.

10. Bere T, Flørenes TW, Nordsletten L, et al. Sex differences in the risk of injury in World Cup alpine skiers: a 6-year cohort study. $\mathrm{Br} J$ Sports Med 2014;48:36-40.

11. Van Ginkel S, Amami M, Dela F, et al. Adjustments of muscle capillarity but not mitochondrial protein with skiing in the elderly. Scand J Med Sci Sports Published Online First: 28 September 2014.

12. LaStayo PC, Pierotti DJ, Pifer J, et al. Eccentric ergometry: increases in locomotor muscle size and strength at low training intensities. $A m$ Physiol Regul Integr Comp Physiol 2000;278:R1282-8.

13. Greig $M$. The influence of soccer-specific fatigue on peak isokinetic torque production of the knee flexors and extensors. Am J Sports Med 2008;36:1403-9.

14. Koller A, Sumann G, Schobersberger W, et al. Decrease in eccentric hamstring strength in runners in the Tirol Speed Marathon. $\mathrm{Br} \mathrm{J}$ Sports Med 2006;40:850-2.

15. Wörndle W. Alpiner Skilauf. In: Österreichischer Skischulverband, ed. Snowsport Austria-Die österreichischen Skischulen. Purkersdorf, Austria: Verlag Brüder Hollinek, 2011:33-138.

16. Schnohr P, O'Keefe JH, Marott JL. Dose of jogging and long-term mortality. J Am Coll Cardiol 2015;65:411-19.

17. Ruedl G, Webhofer M, Helle K, et al. Leg dominance is a risk factor for noncontact anterior cruciate ligament injuries in female recreational skiers. Am J Sports Med 2012;40:1269-73.

18. Bovens AMPM, van Baak MA, Vrencken JGPM, et al. Maximal aerobic power in cycle ergometry in middle-aged men and women, active in sports, in relation to age and physical activity. Int $J$ Sports Med 1993;14:66-71. 
19. Bere T, Bahr R. Injury prevention advances in alpine ski racing: harnessing collaboration with the International Ski Federation (FIS), long-term surveillance and digital technology to benefit athletes. $\mathrm{Br} J$ Sports Med 2014;48:738.

20. Ruedl G, Fink C, Schranz A, et al. Impact of environmental factors on knee injuries in male and female recreational skiers. Scand $J$ Med Sci Sports 2012;22:185-9.

21. Kröll J, Müller E, Seifert JG, et al. Changes in quadriceps muscle activity during sustained recreational alpine skiing. J Sports Sci Med 2011;10:81-92.

22. Hollander DB, Durand RJ, Trynicki JL, et al. RPE, pain, and physiological adjustment to concentric and eccentric contractions. Med Sci Sports Exerc 2003;35:1017-25.

23. Meyer K, Steiner R, Lastayo P, et al. Eccentric exercise in coronary patients: central hemodynamic and metabolic responses. Med Sci Sports Exerc 2003;35:1076-82.

24. Hiemstra LA, Webber S, McDonald PB, et al. Knee strength deficits after hamstring tendon and patellar tendon anterior cruciate ligament reconstruction. Med Sci Sports Exerc 2000;32:1472-9.

25. Jordan MJ, Aagaard P, Herzog W. Rapid hamstrings/quadriceps strength in ACL-reconstructed elite alpine ski racers. Med Sci Sports Exerc 2015;47:109-19.

26. Neumayr G, Hoertnagl H, Pfister R, et al. Physical and physiological factors associated with success in professional alpine skiing. Int $J$ Sports Med 2003;24:571-5.
27. Maffiuletti NA, Impellizzeri F, Rampinini E, et al. Letter to the editor -is aerobic power really critical for success in alpine skiing? Int $J$ Sports Med 2006;27:166-7.

28. Kröll J, Wakeling JM, Seifert JG, et al. Quadriceps muscle function during recreational alpine skiing. Med Sci Sports Exerc 2010;42:1545-56.

29. McCall A, Carling C, Nedelec M, et al. Risk factors, testing and preventive strategies for non-contact injuries in professional football: current perceptions and practices of 44 teams from various premier leagues. Br J Sports Med 2014;48:1352-7.

30. McCall A, Carling C, Davison M, et al. Injury risk factors, screening tests and preventative strategies: a systematic review of evidence that underpins the perceptions and practices of 44 football (soccer) teams from various premier leagues. Br J Sports Med 2015;49:583-9.

31. Barrata R, Solomonow M, Zhou BH, et al. Muscular co-activation. The role of the antagonist musculature in maintaining knee stability. Am J Sports Med 1988;16:113-22.

32. McWilliams BA, Wilson DR, DesJardins JD, et al. Hamstring co-contraction reduces internal rotation, anterior translation, and anterior cruciate ligament load in weight-bearing flexion. J Orthop Res 1999;17:817-22.

33. Thorlund JB, Michalsik LB, Madsen K, et al. Acute fatigue-induced changes in muscle mechanical properties and neuromuscular activity in elite handball players following a handball match. Scand $J$ Med Sci Sports 2008;18:462-72. 